\title{
Qualidade de vida de idosas submetidas ao tratamento de câncer de mama
}

Quality of life of elderly women
undergoing treatment for breast cancer

FisiSenectus. Unochapecó Ano 1, n. 1 - Jan./Jun. 2013 p. $13-24$

\section{Dinara Hansen}

Mestre em Gerontologia Biomédica pela PUCRS, docente da Universidade de Cruz Alta.

\section{Resumo}

Introdução: o câncer de mama é o câncer mais comum entre as mulheres e ocorre em maior número após os 60 anos de idade, sendo idade dependente. 0 tratamento do câncer está voltado para o aumento das possibilidades de cura e sobrevida, bem como melhorar a qualidade de vida dos pacientes. Objetivos: avaliar o impacto do tratamento de câncer de mama na qualidade de vida de um grupo de mulheres após os 60 anos de idade. Materiais e métodos: foi realizado um estudo quantitativo, transversal com enfoque descritivo e analítico em uma amostra de 50 idosas que realizaram tratamento de câncer de mama e um grupo controle de 50 idosas sem o diagnóstico de câncer de mama, frequentadoras do Hospital São Lucas da PUCRS. Resultados: as idades variaram de 60 a 94 anos, com média de 70,76 anos e desvio padrão de 6,95 anos. A qualidade de vida geral do grupo de câncer de mama mostrou-se significativamente melhor comparada ao grupo controle. Na relação da qualidade de vida e dados sociodemográficos, o estado de saúde foi o que mais contribuiu para melhor qualidade de vida. Estado civil e primeiro grau completo também tiveram interferência significativa na qualidade de vida. Conclusão: o tratamento de câncer de mama tem impacto na qualidade de vida na maioria das vezes enquanto está sendo realizado. Após o término do tratamento, a qualidade de vida tende a melhorar.

\section{Palavras-chave}

Câncer de mama. Qualidade de vida. Saúde. 


\begin{abstract}
Introduction: the breast cancer is the most common cancer among women and occurs in greater numbers after 60 years of age, and it is age dependent. The cancer treatment is focused on increasing the opportunities for cure and survival, and improving the quality of life of patients. Objective: evaluate the impact of the treatment of breast cancer in the quality of life of a group of women after 60 years of age. Materials and Methods: this was a quantitative, cross-sectional with observation and analytical study on a sample of 50 elderly women who underwent treatment for breast cancer and a control group of 50 elderly people without a diagnosis of breast cancer, who frequented the St. Luke's Hospital PUCRS. Results: the ages ranged from 60 to 94 years, with an average of 70.76 years and standard deviation of 6.95 years. The quality of life of the general group of breast cancer proved to be significantly better compared to the control group. In respect of quality of life and socio-demographic data, the state of health was the largest contributor to improved quality of life. Marital status and complete the first grade also had significant interference in quality of life. Conclusion: the treatment of breast cancer has an impact on quality of life in most of the time while being held. After the end of treatment, quality of life tends to improve.
\end{abstract}

\title{
Keywords
}

Breast cancer. Quality of life. Health.

\section{Introdução}

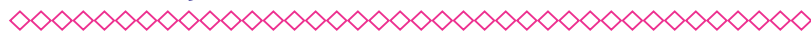

0 câncer de mama é o segundo tipo de câncer mais frequente no mundo e o primeiro entre as muIheres, tanto em países desenvolvidos quanto em países em desenvolvimento. No Brasil, de acordo com as estimativas realizadas pelo Ministério da Saúde, por meio do Instituto Nacional de Câncer (INCA), para o ano de 2012 esperavam-se 52.680 casos novos de câncer da mama, com um risco estimado de 52 casos a cada cem mil mulheres ${ }^{1}$.

Este tipo de câncer é considerado raro antes dos 35 anos de idade, e no Brasil apresenta maior incidência nas mulheres na faixa etária entre $40 \mathrm{e}$ 69 anos de idade ${ }^{1}$. Desta forma, tendo em vista o avanço na estrutura etária nacional, acredita-se na possibilidade do aumento das taxas de incidência desta doença, assim como também dos índices de mortalidade por esse agravo ${ }^{2}$.

O tratamento do câncer de mama está voltado para o aumento da possibilidade de cura e sobrevida, bem como melhorar a Qualidade de Vida (QV) das pacientes. Apesar disso, ele costuma ter efeitos secundários importantes na vida desses pacientes $^{3}$. Por esta razão, nos últimos anos, muitos investigadores têm-se dedicado ao estudo da QV e da adaptação psicossocial da mulher com câncer da mama ${ }^{4-7}$.
Diferentes definições de qualidade de vida têm sido sugeridas em diversos países e diferentes culturas. De acordo com a Organização Mundial de Saúde (OMS), a QV é definida como: "[...] a percepção que o indivíduo tem de sua posição na vida dentro do contexto de sua cultura e do sistema de valores de onde vive, e em relação a seus objetivos, expectativas, padrões e preocupações." ${ }^{8}$

O grupo de especialistas em Qualidade de Vida da Organização Mundial da Saúde (OMS) elaborou um instrumento genérico de avaliação de qualidade de vida, construído por meio de um método transcultural (WHOQOL) $)^{9-11}$.

No Brasil, o Grupo de Estudos em Qualidade de Vida, sob a coordenação do Dr. Marcelo Pio de Almeida Fleck, do Departamento de Psiquiatria e Medicina Legal da Universidade Federal do Rio Grande do Sul, vêm estudando e pesquisando essa temática, contribuindo na validação em Português dos instrumentos WHOQOL-100, WHOQOL-BREF e mais recentemente o WHOQOL-OLD ${ }^{12}$. O presente estudo teve como objetivo avaliar o impacto do tratamento de câncer de mama na QV de idosas.

\section{Materiais e métodos}

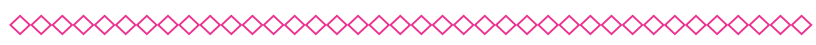

Realizou-se um estudo transversal com enfoque descritivo e analítico em Porto Alegre no pe- 
ríodo entre agosto e novembro de 2008 , no qual a variável de interesse foi a qualidade de vida de idosas que realizaram tratamento de câncer de mama. Foram escolhidos para a realização do estudo os ambulatórios de Ginecologia e de Geriatria do Hospital São Lucas (HSL) da Pontifícia Universidade Católica do Rio Grande do Sul (PUCRS).

Para o cálculo amostral foram utilizados os escores de 0 a 100 para os diferentes domínios dos instrumentos que medem qualidade de vida e foi levado em consideração que estes apresentam média aproximada de 56 e desvio padrão aproximado de 9. Para encontrarmos uma diferença de 6 entre as médias dos dois grupos, considerando-se um erro alfa de 0,05 e um poder de estudo de $90 \%$, é necessário 49 pacientes em cada grupo. Foi utilizado, portanto, uma amostra de cinquenta idosas em cada grupo.

No período entre agosto e novembro de 2008 foram selecionadas cinquenta idosas com diagnóstico de câncer de mama no ambulatório de Ginecologia e cinquenta idosas sem o diagnóstico de câncer de mama, formando o grupo controle, no ambulatório de Geriatria do HSL.

Foram incluídas, no grupo de câncer de mama, mulheres com mais de 60 anos de idade, sem alterações cognitivas, independentes do ponto de vista físico e que realizaram tratamento de câncer de mama, com ou sem reconstrução mamária no HSL-PUCRS há no mínimo seis meses. No grupo controle, foram incluídas mulheres com mais de 60 anos de idade, sem alterações cognitivas, independentes do ponto de vista físico e sem diagnóstico de câncer de mama, que compareceram ao Ambulatório de Geriatria do HSL para realização de consulta de rotina.

Após anuência das pacientes ao consentimento livre e esclarecido, foram aplicados os questionários validados no Brasil e descritos na literatura internacional WHOQOL-BREF e WHOQOL-OLD às pacientes com mais de seis meses de tratamento de câncer de mama e ao grupo controle. Foram coletados também dados sociodemográficos, como idade, estado civil, grau de escolaridade, realização de atividade física e estado de saúde. Após, os dados coletados foram analisados estatisticamente e discutidos.

Os questionários foram aplicados ao grupo de câncer de mama no momento de sua consul- ta de seguimento no Ambulatório de Ginecologia do HSL-PUCRS. A pesquisadora selecionou as pacientes seguindo o agendamento das consultas de cada dia. Eram selecionadas as pacientes com mais de 60 anos de idade e então eram checadas as pastas contendo o diagnóstico, o tipo de tratamento e o tempo de tratamento. Após a checagem, a pesquisadora entrou em contato com as pacientes na sala de espera do ambulatório e explicou os objetivos e métodos envolvidos no trabalho para que pudessem assinar o "Termo de Consentimento Livre e Esclarecido". Os questionários foram aplicados antes da realização da consulta.

Para o grupo controle, escolhido aleatoriamente, a pesquisadora aplicou os questionários no Ambulatório de Geriatria do HSL-PUCRS. As pacientes foram abordadas na sala de espera do ambulatório, onde também foram informadas sobre os objetivos do trabalho para que pudessem assinar o termo de consentimento livre e esclarecido.

Os dados foram coletados diretamente em um banco de dados desenvolvido em Access 2003 e analisados com a utilização do programa SPSS, versão 11.1, autorizado pela PUCRS.

A análise descritiva foi feita por frequência, com os respectivos Índice de Confiança \pm 95\%, medianas, médias e desvios padrões.

As diferenças entre as médias das variáveis quantitativas (diferentes domínios dos questionários de QV) entre os grupos foram avaliadas por meio do Teste t de Student. A avaliação de possíveis vieses de confusão foi avaliada e multivariada.

A associação entre as variáveis quantitativas foi verificada por meio do coeficiente de Correlação de Pearson.

O projeto de pesquisa foi aprovado pelo Comitê de Ética em Pesquisa da Pontifícia Universidade Católica do Rio Grande do Sul e Programa de Pós-graduação em Gerontologia Biomédica pelo ofício CEP 08/04111.

\section{Resultados}

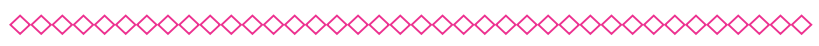

A média de idade entre ambos os grupos foi de 70,76 anos, com um desvio padrão de 6,95. No grupo com diagnóstico de câncer de mama a média 
de idade foi de 69,56 anos, com desvio padrão de 5,8 anos, e no grupo sem diagnóstico foi de 71,96 anos, com desvio padrão de 7,8 anos.

A faixa etária de maior incidência dentre os grupos foi a dos 60-69 anos de idade, com $48 \%$. No grupo de câncer prevaleceu a faixa etária de 60-69 anos (56\%) e no grupo controle prevaleceu a faixa etária de 70-79 anos (42\%). A faixa etária apresentou diferença significativa entre os dois grupos com $p=0,042$.

Em relação a percepção de saúde de ambos os grupos, $44 \%$ das idosas referiram estado de saúde nem ruim nem bom; $45 \%$ referiram estado de saúde bom e muito bom; e $11 \%$ referiram estado de saúde fraco e ruim. No grupo de câncer de mama a maioria, 52\% das idosas, referiu estado de saúde bom/muito bom e no grupo controle, a maioria, $44 \%$ das idosas, referiu estado de saúde nem ruim nem bom. Houve, nesta variável, diferença significativa entre os grupos com $p=0,037$, sendo o grupo de câncer de mama o que referiu melhor estado de saúde.

Sobre a realização de atividade física, 56\% das idosas não realizavam nenhuma atividade física, $33 \%$ realizavam numa frequência de até três vezes/semana e $11 \%$ realizavam mais de três vezes/ semana.

Referente ao estado civil de ambos os grupos, $17 \%$ são solteiras, separadas ou divorciadas, $45 \%$ são casadas e $38 \%$ viúvas. A percentagem de viúvas, tanto no grupo de câncer de mama quanto no grupo controle, foi de 38\%, representando 19 idosas em cada grupo. Estes dados podem ser melhor visualizados na tabela 1 .

A QV total do grupo de câncer de mama no WHOQOL-BREF foi de 73,7, com desvio padrão de 10,8 , e no grupo controle foi de 72,7 , com desvio padrão de 12,4 . Não houve diferença significativa entre os grupos nesta variável.

Nos domínios do WHOQOL-BREF, houve diferença significativa somente entre as variáveis do geral entre os domínios com $p=0,030$ e na questão 1 com $p=0,030$, sendo que em ambos o grupo de câncer de mama apresentou valores superiores ao grupo controle (tabela 2).

A QV total do grupo de câncer de mama no WHOQOL-OLD foi de 79,0, com desvio padrão de
10,4 , e do grupo controle foi de 76,4 , com desvio padrão de 10,0 . Não houve diferença significativa nesta variável.

Nas facetas do WHOQOL-OLD houve diferença significativa somente no Funcionamento Sensório $(p=0,015)$, que se refere às perdas na audição, visão, paladar, olfato ou tato, com repercussão na vida diária, interferência na participação em algumas atividades ou na habilidade em interagir com outras pessoas; o grupo de câncer obteve média de $82,3( \pm 16,3)$ e o grupo controle $72,1( \pm 24,0)$.

Foi feita uma análise de regressão linear considerando a idade das idosas. Não houve alteração significativa na QV ajustando por idade.

Os escores médios e desvio padrão nos quatro domínios do WHOQOL-BREF e nas seis facetas do WHOQOL-OLD de ambos os grupos foram comparados com os dados sociodemográficos.

A tabela 3 apresenta a relação entre WHOQOL-BREF e WHOQOL-OLD e os dados sociodemográficos estado civil, primeiro grau completo e faixa etária.

No que ser refere ao estado civil de ambos os grupos, foi encontrada diferença significativa somente na relação com o escore do WHOQOL-OLD na faceta intimidade, $p=0,032$, sendo que o grupo de idosas casadas apresentou melhor QV na faceta intimidade, com valor de 85,4 e desvio padrão de 12,5 , enquanto que o grupo de viúvas apresentou menor qualidade de vida nesta faceta, com valor de 77,9 e desvio padrão de 11,7 . As idosas separadas, solteiras e divorciadas tiveram valor intermediário na qualidade de vida nesta mesma faceta $(81,2 \pm 14,2)$.

Completar o primeiro grau apresentou diferença significativa somente na relação com o escore do WHOQOL-BREF no domínio social, com $\mathrm{p}=0,028$. 0 grupo de idosas que completou o primeiro grau apresentou valor de 82,0 e desvio padrão de 12,5, enquanto que o grupo que não completou o primeiro grau apresentou um valor menor, de 76,0, e desvio padrão de 13,0.

A relação da faixa etária com o WHOQOL-BREF mostrou diferença significativa na questão $1(p=$ 0,054), em que idosas na faixa etária dos 70-79 anos apresentaram maior valor. Na relação com o WHOQOL-OLD houve diferença significativa 
somente na faceta do funcionamento sensório $(p=0,009)$, sendo que o grupo da faixa etária entre 60-69 apresentou maior valor. Nas demais facetas e domínios não houve diferença significativa entre as idosas.

Na tabela 4 podem ser visualizados os dados sobre a relação entre estado de saúde e prática de atividade física com os domínios e facetas do WHOQOL-BREF e WHOQOL-OLD.

0 estado de saúde interferiu significativamente na QV das idosas em ambos os questionários. Em relação ao WHOQOL-BREF não houve diferença significativa somente na questão $1(p=0,426)$. Os demais domínios apresentaram diferença significativa entre as idosas.

A realização ou não de atividade física não apresentou diferença significativa entre os grupos tanto na relação dos domínios WHOQOL-BREF quanto com as facetas WHOQOL-OLD.

\section{Discussão}

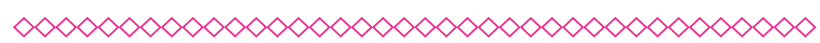

A média de idade entre ambos os grupos deste estudo foi de 70,76 anos, com um desvio padrão de 6,95 anos, e a faixa etária que prevaleceu entre os grupos foi a de 60-69 anos (48\%), estando estas na faixa etária de maior incidência de câncer de mama, segundo o INCA ${ }^{1}$.

Quanto ao fator de escolaridade, mais da metade, 64 (64\%) das entrevistadas relataram não ter concluído o ensino fundamental. Segundo o IBGE, em 2008 a média de anos de estudo das mulheres foi de 7,2 anos, sendo que na área urbana a média era 9,2 anos e na área rural 5,2 anos de estudo. A escolaridade dos idosos brasileiros é ainda considerada baixa: 30,7\% tinham, em 2009, menos de um ano de instrução ${ }^{13}$.

Um estudo realizado no município de Botucatu, em São Paulo, observou que mulheres idosas e mulheres com baixo grau de escolaridade apresentaram menores oportunidades de diagnóstico precoce de câncer de mama, sendo este um dos fatores para o diagnóstico da doença em estádios mais avançados após os 60 anos de idade ${ }^{14}$.

Os resultados do presente estudo mostraram uma diferença significativa no estado de saúde dos grupos, já que a maioria das idosas do grupo de câncer de mama referiu estado de saúde meIhor em relação ao grupo controle. Isto pode ser justificado pelo fato de as idosas do grupo de câncer de mama já terem completado o tratamento e estarem livres da doença.

Um estudo realizado com dados de 2.135 idosos teve como objetivo investigar a influência de determinantes demográficos e socioeconômicos, das doenças crônicas e da capacidade funcional sobre a autopercepção de saúde entre idosos do município de São Paulo. Este estudo mostrou que na ausência de doenças crônicas, ou na presença de duas ou mais doenças crônicas, as mulheres idosas tiveram maior probabilidade de relatar uma boa autopercepção de saúde, principalmente pelo fato de a doença estar sob controle ${ }^{15}$.

Este mesmo estudo também evidenciou que a idade é um importante determinante da autopercepção de saúde. As chances de os idosos relatarem uma saúde negativa foram maiores do que na categoria de referência em todos os grupos etários, mas declinaram com o avançar da idade ${ }^{15}$.

Estes dados citados acima podem justificar o resultado do estado de saúde do grupo controle, obtido no presente estudo, que foi em sua maioria considerado inferior ao do grupo de câncer de mama.

$\mathrm{Na}$ avaliação da QV geral entre ambos os grupos, o grupo de câncer de mama apresentou significativa melhora na QV em relação ao grupo controle. Mesmo assim, os valores apresentados pelo grupo controle identificam uma boa QV.

Um estudo que comparou a prevalência de sintomas climatéricos, a proporção de mulheres com atividade sexual e a QV em mulheres com e sem câncer de mama constatou que mulheres com câncer de mama relataram melhor QV em relação àquelas sem câncer de mama, principalmente em relação a capacidade funcional ${ }^{16}$.

Neste, a QV apresentou mediana dos escores de 90 para o grupo com câncer e de 75 para o grupo sem câncer $(p<0,01)$. A prevalência de sintomas climatéricos foi similar em mulheres com e sem câncer de mama. Foram identificados fatores que se associaram negativamente à QV e que representam eventos comuns na vida da mulher (sintomas climatéricos, ser casada, pós-menopausa) ${ }^{17}$. 
No WHOQOL-BREF houve diferença significativa entre os grupos somente no escore geral dos quatro domínios, sendo que o grupo de câncer de mama apresentou valores maiores em relação ao grupo controle. Já no WHOQOL-OLD houve diferença significativa somente no funcionamento sensório, onde, também, o grupo de câncer apresentou valores mais elevados em relação ao grupo controle.

Um estudo apresentou os resultados da avaliação da qualidade de vida de 294 sobreviventes de câncer de mama. As mulheres avaliaram sua qualidade de vida após o tratamento. A maioria delas considerou boa ou ótima, atribuindo principalmente à saúde pessoal, fé em Deus, bom relacionamento familiar e social, mais valor à vida, entre outros. As demais avaliaram sua qualidade de vida como ruim ou regular, ao que elas atribuíram o medo de recidiva, limitação das atividades, idade, problemas financeiros, entre outros ${ }^{18}$.

Um maior número de mulheres avaliou a qualidade de vida como boa ou ótima ${ }^{18}$, avaliação também obtida por Lindley et al. ${ }^{19}$

Algumas justificativas coincidem com outros trabalhos: bom relacionamento e apoio, saúde física, fé e valorização da vida. As razões indicadas para a avaliação de qualidade de vida ruim ou regular também são citadas em outros trabalhos: dor, insônia, dificuldades psicológicas, preocupações financeiras e medo de recorrência ${ }^{18}$.

0 estado civil apresentou diferença significativa somente na faceta Intimidade do WHOQOL-OLD. A QV foi mais positiva no grupo de idosas casadas em relação aos grupos de solteiras, separadas, divorciadas e viúvas.

Um estudo que avaliou a concordância da percepção da QV entre mulheres com câncer de mama e seus parceiros mostrou que este é o mais frequente cuidador formal da paciente com câncer de mama, sendo uma fonte valiosa de suporte emocional $^{20}$. 0 mesmo estudo mostrou que a existência de um companheiro é um indicador de suporte social que mostra papel importante na sobrevida e na adaptação à doença. Identificou também que a ausência de uma relação de intimidade com o marido ou o namorado não é compensada por uma relação de confiança com um amigo ou membro da família. Isto se deve ao fato de que, na presença de uma doença como o câncer de mama, o marido pode ter influência positiva no que se referente ao estado psicológico da paciente ${ }^{20}$.

A conclusão do primeiro grau de ensino interferiu de forma significativa somente no domínio social do WHOQOL-BREF. Neste domínio, as idosas que não concluíram o primeiro grau apresentaram menor QV comparado ao grupo que concluiu o primeiro grau.

Sprangers et al. ${ }^{21}$ mostraram que fatores como idade avançada, sexo feminino, baixo nível de escolaridade e não ter companheiro estão relacionados a baixos níveis de QV quando relacionados aos domínios do WHOQOL-BREF.

A faixa etária das idosas interferiu de forma significativa no funcionamento sensório do WHOQOL-OLD, sendo que as idosas na faixa etária entre 60-69 anos apresentaram escores maiores em relação às demais faixas etárias. As idosas com mais de 70 anos apresentaram menores valores na QV, podendo ser justificado pelas diversas alterações fisiológicas que ocorrem durante o processo de envelhecimento.

Um estudo que teve como objetivo comparar a QV entre um grupo de idosas e um grupo de mulheres jovens que tiveram câncer de mama mostrou que as idosas tiveram um escore no SF-36 menor do que as mulheres mais jovens, principalmente nas medidas de humor, fadiga e QV relacionada à saúde. Estas idosas mostraram índices de menor QV podendo isto estar relacionado às múltiplas deficiências comuns na população geriátrica ${ }^{22}$.

Com relação ao estado de saúde, o presente estudo mostrou que existe influência deste na QV de ambos os grupos. Quanto melhor o estado de saúde, melhor a QV referida pelas idosas.

No presente estudo, a realização de atividade física não interferiu de forma significativa na relação com a QV. Houve uma tendência de significância no domínio psicológico do WHOQOL-BREF e na QV total do WHOQOL-OLD. Talvez se o número da amostra utilizada fosse maior, a realização de atividade física poderia interferir de forma significativa na melhor QV.

Um estudo que comparou a QV entre mulheres pós-câncer de mama, ativas e inativas, justifica este resultado. No estudo, ser ativa ou sedentária não interferiu de forma significativa na QV ${ }^{23}$. 
Alguns autores afirmam que mulheres portadoras de câncer mais ativas parecem ter uma melhor qualidade de vida do que aquelas menos ativas, mas seus resultados não são capazes de mostrar se esses benefícios provêm de uma meIhoria nas capacidades físicas ou de outros fatores associados ao exercício ${ }^{24}$.

\section{Conclusão}

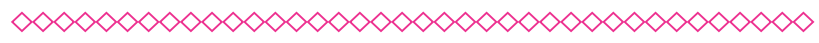

Com este estudo conclui-se que o tratamento do câncer de mama não interfere de forma negativa na QV de idosas após, no mínimo, seis meses de tratamento. Salienta-se que o impacto do tratamento é sentido em maior proporção pelas pacientes mais jovens em função das alterações na sexualidade e na percepção da imagem corporal.

Mulheres com mais de 60 anos acometidas pelo câncer de mama reagem ao diagnóstico da doença e ao tratamento de maneira mais positiva, principalmente em função das experiências já vividas, do bom relacionamento familiar, da melhora da saúde física, da fé e da valorização da vida.

Salienta-se a necessidade de maiores e mais detalhadas investigações sobre o tratamento do câncer de mama e sua influência na QV da população idosa.

\section{Referências}

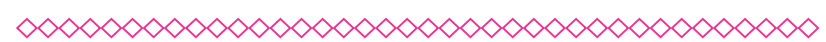

\section{Instituto Nacional de Câncer José Alencar} Gomes da Silva, Coordenação Geral de Ações Estratégicas. Coordenação de Prevenção e Vigilância. Estimativa 2012: incidência de câncer no Brasil. Rio de Janeiro (RJ); 201.

\section{Basílio DV, Mattos IE. Câncer em mulheres} idosas das regiões sul e sudeste do Brasil: evolução da mortalidade no período de 19802005. Rev Bras Epidemiol. 2008;11(2):204-14.

3. Aguiar ADF; Padulha KM, Volpi PTM, Gomes JC, Tartarotti EA,Oliveira MS, et al. Perfil sociodemográfico e clínico das pacientes em tratamento do câncer mamário. Rev Inst Ciênc Saúde. 2008;26(2):191-5.
4. Carver CS, Smith RG, Petronis VM, Antoni MH. Quality of Life Among Long-Term Survivors of Breast Cancer: Different Types of Antecedents Predict Different Classes of Outcomes. PsychoOncology. 2006;15(9):749-758.

5. Helgeson VS, Tomich PL. Surviving cancer: a comparison of 5-year disease-free breast cancer survivors with healthy women. Psycho-Oncology. 2005; 14(4):307-317.

6. Knobf MT. Psychosocial responses in breast cancer survivors. Semin Oncol Nurs 2007;23(1):71-83.

7. Kornblith AB, Powell M, Regan MM, Bennett $S$, Krasner C, Moy B, et al. Long-term psychosocial adjustment of older vs younger survivors of breast and endometrial cancer. Psycho-Oncology. 2007;16:895-903.

8. Campo MO, Rodrigues Neto JFR. Qualidade de vida, um instrumento para promoção da saúde. Rev Baiana Saúde Pública. 2008;32(2):232-40.

9. Veras R. Envelhecimento populacional e as informações de saúde do PNAD: demandas e desafios contemporâneos. Introdução. Cad. Saúde Pública. 2007; 23(10):2463-6.

10. Fleck, MPA; Leal OF, Louzada S, Xavier M, Chachamovich E, Vieira G, et al. Desenvolvimento da versão em português do instrumento de avaliação de qualidade de vida da OMS (WHOQOL-100). Rev Bras Psiquiatr. 1999;21(1):19-28.

11. Paschoal SMP. Qualidade de vida do idoso: construção de um instrumento de avaliação através do método do impacto clínico [tese]. São Paulo: Faculdade de Medicina da USP; 2005.

12. Fleck MPA, Chachamovich E, Trentini CM. Projeto WHOQOL-OLD: método e resultados de grupos focais no Brasil. Rev. Saúde Pública. 2003;37(6).

13. Instituto Brasileiro de Geografia e Estatística. Síntese de indicadores sociais: uma análise das condições de vida da população brasileira 2010 [acesso em 10/11/2011]. Disponível em: http://www.ibge.gov.br/home/estatistica/ populacao/condicaodevida/indicadoresminimos/ sinteseindicsociais2010/SIS_2010.pdf. 
14. Molina L, Dallen I, De LUCA LA. Análise das oportunidades de diagnóstico precoce para as neoplasias malignas de mama. Rev Assoc Méd Bras. 2003;49(2):185-90. In: Guerra MR, Gallo CVM, Mendonça GAS. Risco de câncer no Brasil: tendências e estudos epidemiológicos mais recentes. Rev. bras. cancerol, 2005.

15. Alves LC, Rodrigues RN. Determinantes da autopercepção de saúde entre idosos do Município de São Paulo, Brasil. Rev Panam Salud Pub. 2005;17(5).

16. Vicini FA, Recht A. Age at diagnosis and outcome for woman with ductal carcinoma-in-situ of the breast: a critical review of the literature. J. Clin. Oncol. 2002; 20(11):2736-44.

17. Conde DM. Câncer de mama, menopausa e qualidade de Vida. Rev Bras Ginecol Obstet. 2005;27(8):502.

18. Dow KH, Ferrell BR, Leigh S, Ly J, Gulasekaram P. An evaluation of the quality of life among longterm survivors of breast cancer. Breast cancer res treat. 1996;39(3):261-273.

19. Lindley C, Vasa S, Sawyer WT, Winer EP. Quality of life and preferences for treatment following systematic adjuvant therapy for earlystage breast cancer. J Clin Oncol. 1998;16(4):1380-1387.
20. Rabin EG, Heldt E, Hirakata VN, Fleck MP. Quality of life predictors in breast cancer women. EUR Journal oncology nursing.2008;12(1):53-57.

21. Sprangers MA, De Regt EB, Andries F, Van Agt HM, Bijl RV, Boer JB, et al. Which chronic conditions are associated with better or poorer quality of life? J Clin Epidemiol. 2000;53(9):895-907.

22. Robb C, Haley WE, Balducci L, Extermann M, Perkins E, Small B, et al. Impacto of breast câncer survivorship on quality of life in older women. Crit. Rev Oncol Hematol. 2007;62: 84-91

23. Feuser AF, Aguiar Junior AS. Efeitos do estilo de vida sedentário na qualidade de vida em mulheres pós - câncer de mama no Vale do Braço Norte[periódico na internet]. 2005 [acesso em 14/10/2011]. Disponível em http://www. fisio-tb.unisul.br/Tccs/07a/alessandra/artigo_ alessandra.pdf.

24. Schneider CM, Hsieh CC, Sprod LK, Carter SD, Hayward R. Cancer treatment-induced alterations in muscular fitness and quality of life: the role of exercise training. Ann Oncol. 2007; 18(12):1957-1962. 


\section{Anexos}

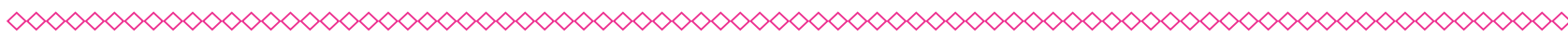

Tabela 1 - Dados sociodemográficos

\begin{tabular}{|c|c|c|c|c|}
\hline Dados & $\begin{array}{l}\text { Grupo CA Mama } \\
\text { n (\%) }\end{array}$ & $\begin{array}{l}\text { Grupo Controle } \\
\text { n (\%) }\end{array}$ & $\begin{array}{l}\text { Geral } \\
\mathrm{n}(\%)\end{array}$ & $\mathbf{P}$ \\
\hline \multicolumn{5}{|l|}{ Faixa etária } \\
\hline $60-69$ & $28(56)$ & $20(40)$ & $48(48)$ & $0,042 *$ \\
\hline $70-79$ & $19(38)$ & $21(42)$ & $40(40)$ & \\
\hline 80 ou mais & $3(6)$ & $9(18)$ & $12(12)$ & \\
\hline \multicolumn{5}{|l|}{$1^{\circ}$ Grau completo } \\
\hline Sim & $16(32)$ & $20(40)$ & $36(36)$ & $0,405 * *$ \\
\hline Não & $34(68)$ & $30(60)$ & $64(64)$ & \\
\hline \multicolumn{5}{|l|}{ Estado de saúde } \\
\hline Ruim / Fraco & $2(4)$ & $9(18)$ & $11(11)$ & $0,037 *$ \\
\hline Nem ruim nem bom & $22(44)$ & $22(44)$ & $44(44)$ & \\
\hline Bom / Muito bom & $26(52)$ & $19(38)$ & $45(45)$ & \\
\hline \multicolumn{5}{|l|}{ Atividade física } \\
\hline Nenhuma & $28(56)$ & $28(56)$ & $56(56)$ & $0,662 *$ \\
\hline Até 3 vezes/semana & $18(36)$ & $15(30)$ & $33(33)$ & \\
\hline Mais 3 vezes/semana & $4(8)$ & $7(14)$ & $11(11)$ & \\
\hline \multicolumn{5}{|l|}{ Estado civil } \\
\hline Solteira/separada/divorciada & $8(16)$ & $9(18)$ & $17(17)$ & $0,960 * *$ \\
\hline Viúva & $19(38)$ & $19(38)$ & $38(38)$ & \\
\hline Casada & $23(46)$ & $22(44)$ & $45(45)$ & \\
\hline
\end{tabular}

Legenda: * Tendência linear. ** Correlação de Pearson

Fonte: elaboração da autora.

(clique para voltar ao texto) 
Tabela 2 - Resultado dos domínios do WHOQOL-BREF e facetas do WHOQOL-OLD relacionado ao câncer de mama

\begin{tabular}{lcccc} 
Variável & $\begin{array}{c}\text { Grupo (A mama } \\
\text { M }( \pm \mathrm{DP})\end{array}$ & $\begin{array}{c}\text { Grupo Controle } \\
\mathrm{M}( \pm \mathrm{DP})\end{array}$ & $\mathbf{P}$ & \\
Whoqol-bref & & & & \\
Q1 & $76,5 \pm 15,4$ & $73,0 \pm 15,0$ & 0,254 & 0,250 \\
Q2 & $69,5 \pm 18,4$ & $59,5 \pm 26,1$ & $\mathbf{0 , 0 3 0}$ & $\mathbf{0 , 0 3 1}$ \\
D Geral & $73,0 \pm 13,4$ & $66,2 \pm 16,9$ & $\mathbf{0 , 0 3 0}$ & $\mathbf{0 , 0 3 1}$ \\
D físico & $67,9 \pm 15,3$ & $61,8 \pm 17,8$ & 0,072 & 0,134 \\
D psicológico & $76,2 \pm 13,1$ & $76,3 \pm 14,2$ & 0,976 & 0,931 \\
D ambiental & $74,6 \pm 10,6$ & $77,3 \pm 17,5$ & 0,362 & 0,339 \\
D social & $76,8 \pm 13,4$ & $79,6 \pm 12,7$ & 0,286 & 0,187 \\
Total & $73,7 \pm 10,8$ & $72,7 \pm 12,4$ & 0,681 & 0,858 \\
Whoqol-old & & & & \\
FS & $82,3 \pm 16,3$ & $72,1 \pm 24,0$ & $\mathbf{0 , 0 1 5}$ & $\mathbf{0 , 0 5 7}$ \\
AUT & $78,1 \pm 17,0$ & $76,0 \pm 17,4$ & 0,540 & 0,363 \\
PPF & $73,7 \pm 13,0$ & $74,6 \pm 12,0$ & 0,727 & 0,910 \\
PS & $75,2 \pm 15,9$ & $76,8 \pm 15,2$ & 0,604 & 0,383 \\
MEM & $80,0 \pm 17,9$ & $77,0 \pm 21,8$ & 0,455 & 0,454 \\
INT & $81,2 \pm 11,8$ & $81,7 \pm 14,1$ & 0,848 & 0,946 \\
Total & $79,0 \pm 10,4$ & $76,4 \pm 10,0$ & 0,193 & 0,354 \\
\hline
\end{tabular}

* Regressão linear por idade Fonte: elaboração da autora. 
Tabela 3 - Resultado dos domínios do WHOQOL-BREF e facetas do WHOQOL-OLD relacionado ao estado civil, $1^{\circ}$ grau completo e faixa etária

\begin{tabular}{|c|c|c|c|c|c|c|c|c|c|c|c|}
\hline \multirow[t]{2}{*}{ Variáveis } & \multicolumn{4}{|c|}{ Estado civil } & \multicolumn{3}{|c|}{$1^{\circ}$ grau completo } & \multicolumn{4}{|c|}{ Faixa etária } \\
\hline & $\begin{array}{l}\text { Solteira } \\
\text { M ( } \pm D P)\end{array}$ & $\begin{array}{l}\text { Casada } \\
M( \pm D P)\end{array}$ & $\begin{array}{l}\text { Viúva } \\
\text { M (土DP) }\end{array}$ & $\mathbf{P}$ & $\operatorname{Sim}_{M( \pm D P)}$ & $\begin{array}{c}\text { Não } \\
\text { M (土DP) }\end{array}$ & $\mathbf{P}$ & $\begin{array}{c}60-69 \\
M( \pm D P)\end{array}$ & $\begin{array}{c}70-79 \\
M( \pm D P)\end{array}$ & $\begin{array}{l}80 \text { ou }+ \\
M( \pm D P)\end{array}$ & $\mathbf{P}$ \\
\hline \multicolumn{12}{|l|}{ W-BREF } \\
\hline Questão 1 & $73,5 \pm 13,8$ & $74,4 \pm 15,5$ & $75,6 \pm 15,9$ & 0,880 & $75,0 \pm 14,6$ & $74,6 \pm 15,7$ & 0,901 & $74,4 \pm 15,8$ & $74,3 \pm 16,4$ & $77,0 \pm 7,2$ & 0,855 \\
\hline Questão 2 & $66,1 \pm 29,2$ & $64,4 \pm 22,9$ & $63,8 \pm 20,7$ & 0,941 & $66,6 \pm 26,0$ & $63,2 \pm 21,3$ & 0,509 & $61,9 \pm 25,2$ & $70,6 \pm 17,8$ & $54,1 \pm 25,7$ & 0,054 \\
\hline Geral & $69,8 \pm 19,2$ & $69,4 \pm 16,3$ & $69,7 \pm 13,2$ & 0,994 & $70,8 \pm 16,9$ & $68,9 \pm 14,9$ & 0,578 & $68,2 \pm 17,6$ & $72,5 \pm 13,6$ & $65,6 \pm 12,0$ & 0,285 \\
\hline Físico & $71,8 \pm 16,5$ & $62,7 \pm 18,4$ & $64,2 \pm 14,4$ & 0,161 & $67,2 \pm 19,7$ & $63,3 \pm 14,9$ & 0,333 & $66,5 \pm 18,9$ & $64,7 \pm 15,6$ & $58,9 \pm 9,8$ & 0,380 \\
\hline Psicológico & $81,3 \pm 12,9$ & $74,3 \pm 13,7$ & $76,3 \pm 13,4$ & 0,195 & $78,4 \pm 16,0$ & $75,0 \pm 11,9$ & 0,271 & $75,9 \pm 16,4$ & $76,6 \pm 10,5$ & $76,3 \pm 10,4$ & 0,971 \\
\hline Ambiental & $76,9 \pm 13,6$ & $75,7 \pm 15,3$ & $75,8 \pm 14,2$ & 0,956 & $79,1 \pm 14,2$ & $74,2 \pm 14,4$ & 0,102 & $76,7 \pm 14,0$ & $74,7 \pm 15,8$ & $77,0 \pm 12,3$ & 0,795 \\
\hline Social & $80,6 \pm 13,5$ & $77,2 \pm 13,7$ & $78,2 \pm 12,4$ & 0,664 & $82,0 \pm 12,5$ & $76,0 \pm 13,0$ & 0,028 & $79,1 \pm 12,9$ & $78,6 \pm 13,8$ & $73,1 \pm 11,1$ & 0,365 \\
\hline Total & $77,2 \pm 12,0$ & $71,9 \pm 11,9$ & $73,1 \pm 10,9$ & 0,280 & $76,0 \pm 12,9$ & $71,7 \pm 10,5$ & 0,092 & $73,8 \pm 13,0$ & $73,5 \pm 10,8$ & $69,9 \pm 7,0$ & 0,573 \\
\hline \multicolumn{12}{|l|}{ W-OLD } \\
\hline FS & $80,0 \pm 26,6$ & $77,3 \pm 20,1$ & $75,1 \pm 20,6$ & 0,742 & $75,6 \pm 23,7$ & $77,7 \pm 19,9$ & 0,662 & $82,0 \pm 20,0$ & $75,6 \pm 19,3$ & $61,4 \pm 25,6$ & 0,009 \\
\hline AUT & $84,7 \pm 15,8$ & $75,5 \pm 17,7$ & $77,9 \pm 15,6$ & 0,171 & $76,2 \pm 18,5$ & $78,9 \pm 15,7$ & 0,445 & $80,5 \pm 16,1$ & $75,1 \pm 17,4$ & $76,5 \pm 17,2$ & 0,323 \\
\hline PPF & $75,3 \pm 14,5$ & $74,0 \pm 12,0$ & $75,3 \pm 12,2$ & 0,873 & $76,0 \pm 14,2$ & $73,9 \pm 11,2$ & 0,461 & $74,6 \pm 13,5$ & $74,6 \pm 10,9$ & $75,5 \pm 13,1$ & 0,974 \\
\hline PS & $76,9 \pm 17,9$ & $77,3 \pm 15,6$ & $74,6 \pm 15,1$ & 0,736 & $79,3 \pm 16,9$ & $74,4 \pm 14,8$ & 0,159 & $78,1 \pm 16,4$ & $76,3 \pm 15,8$ & $68,7 \pm 10,6$ & 0,180 \\
\hline MEM & $79,6 \pm 20,3$ & $79,0 \pm 22,6$ & $76,9 \pm 16,7$ & 0,860 & $79,3 \pm 22,4$ & $77,7 \pm 18,7$ & 0,724 & $75,6 \pm 23,8$ & $81,0 \pm 14,9$ & $80,2 \pm 18,0$ & 0,443 \\
\hline INT & $81,2 \pm 14,2$ & $85,4 \pm 12,5$ & $77,9 \pm 11,7$ & 0,032 & $84,3 \pm 12,6$ & $80,5 \pm 12,9$ & 0,155 & $82,5 \pm 13,0$ & $82,5 \pm 12,3$ & $77,6 \pm 13,9$ & 0,462 \\
\hline Total & $79,6 \pm 11,5$ & $78,1 \pm 9,8$ & $76,3 \pm 10,3$ & 0,526 & $78,5 \pm 12,3$ & $77,2 \pm 8,9$ & 0,599 & $78,9 \pm 11,3$ & $77,5 \pm 9,1$ & $73,3 \pm 8,9$ & 0,246 \\
\hline
\end{tabular}

FS = funcionamento sensório; $A U T$ = autonomia; PPF = atividades passadas, presentes e futuras; PS = participação social; $M E M=$ morte e morrer; $I N T$ = intimidade Fonte: elaboração da autora. 
Tabela 4 - Resultado dos domínios do WHOQOL-BREF e facetas do WHOQOL-OLD relacionado ao estado de saúde e atividade física

\begin{tabular}{|c|c|c|c|c|c|c|c|c|}
\hline \multirow[t]{2}{*}{ Variáveis } & \multicolumn{4}{|c|}{ Estado de saúde } & \multicolumn{4}{|c|}{ Atividade física } \\
\hline & $\begin{array}{c}\text { R-F } \\
M( \pm D P)\end{array}$ & $\begin{array}{l}\text { NR-NB } \\
M( \pm D P)\end{array}$ & $\begin{array}{c}\text { B-MB } \\
M( \pm D P)\end{array}$ & $\mathbf{p}$ & $\begin{array}{c}N \\
( \pm D P)\end{array}$ & $\begin{array}{c}\text { Até3x/semana } \\
M( \pm D P)\end{array}$ & $\begin{array}{c}+3 x / \text { semana } \\
M( \pm D P)\end{array}$ & $\mathbf{p}$ \\
\hline \multicolumn{9}{|l|}{ W-BREF } \\
\hline Q1 & $70,4 \pm 18,7$ & $73,8 \pm 17,0$ & $76,6 \pm 12,3$ & 0,426 & $74,5 \pm 14,6$ & $75,0 \pm 16,5$ & $75,0 \pm 15,8$ & 0,990 \\
\hline Q2 & $34,0 \pm 23,1$ & $58,5 \pm 20,8$ & $77,7 \pm 14,3$ & 0,000 & $63,8 \pm 23,3$ & $65,9 \pm 22,3$ & $63,6 \pm 25,8$ & 0,914 \\
\hline Geral & $52,2 \pm 13,4$ & $66,1 \pm 14,9$ & $77,2 \pm 12,0$ & 0,000 & $69,1 \pm 15,6$ & $70,4 \pm 15,5$ & $69,3 \pm 17,1$ & 0,934 \\
\hline Físico & $49,3 \pm 15,4$ & $60,5 \pm 16,4$ & $72,9 \pm 13,2$ & 0,000 & $64,0 \pm 16,2$ & $64,6 \pm 16,8$ & $70,1 \pm 20,6$ & 0,549 \\
\hline Psicológico & $66,6 \pm 19,0$ & $74,1 \pm 13,1$ & $80,7 \pm 10,7$ & 0,003 & $74,0 \pm 14,5$ & $77,6 \pm 11,9$ & $83,7 \pm 10,6$ & 0,075 \\
\hline Ambiental & $67,4 \pm 18,8$ & $75,1 \pm 16,5$ & $78,8 \pm 9,9$ & 0,005 & $75,0 \pm 15,9$ & $75,2 \pm 12,5$ & $83,3 \pm 10,5$ & 0,207 \\
\hline Social & $68,7 \pm 10,5$ & $76,9 \pm 13,2$ & $81,7 \pm 12,3$ & 0,008 & $77,2 \pm 13,6$ & $79,3 \pm 11,6$ & $79,5 \pm 15,2$ & 0,730 \\
\hline Total & $61,6 \pm 11,0$ & $70,8 \pm 11,0$ & $78,4 \pm 9,4$ & 0,000 & $72,0 \pm 11,7$ & $73,8 \pm 10,9$ & $77,6 \pm 12,8$ & 0,335 \\
\hline \multicolumn{9}{|l|}{ W-OLD } \\
\hline FS & $57,9 \pm 24,6$ & $74,5 \pm 19,2$ & $84,0 \pm 19,2$ & 0,001 & $73,2 \pm 22,7$ & $82,6 \pm 16,2$ & $79,5 \pm 24,5$ & 0,136 \\
\hline AUT & $75,0 \pm 17,4$ & $75,8 \pm 17,8$ & $80,6 \pm 15,5$ & 0,348 & $76,2 \pm 15,1$ & $77,6 \pm 17,9$ & $87,5 \pm 19,7$ & 0,127 \\
\hline PPF & $65,3 \pm 12,6$ & $75,5 \pm 11,7$ & $76,2 \pm 12,2$ & 0,026 & $74,2 \pm 12,7$ & $73,3 \pm 11,0$ & $81,2 \pm 13,4$ & 0,176 \\
\hline PS & $72,7 \pm 13,1$ & $75,1 \pm 15,7$ & $78,2 \pm 16,4$ & 0,483 & $74,2 \pm 15,9$ & $77,4 \pm 15,3$ & $83,5 \pm 14,8$ & 0,180 \\
\hline MEM & $64,7 \pm 27,8$ & $81,2 \pm 13,5$ & $78,9 \pm 22,1$ & 0,049 & $75,6 \pm 19,7$ & $83,8 \pm 18,8$ & $76,1 \pm 23,5$ & 0,179 \\
\hline INT & $73,2 \pm 14,5$ & $82,0 \pm 13,0$ & $85,7 \pm 10,9$ & 0,007 & $80,7 \pm 13,0$ & $82,8 \pm 12,0$ & $85,2 \pm 14,5$ & 0,524 \\
\hline Total & $68,1 \pm 11,2$ & $77,1 \pm 9,1$ & $80,6 \pm 9,7$ & 0,001 & $75,7 \pm 10,4$ & $79,6 \pm 9,1$ & $82,1 \pm 11,5$ & 0,074 \\
\hline
\end{tabular}

FS = funcionamento sensório; $A U T$ = autonomia; PPF = atividades passadas, presentes e futuras; PS = participação social; $M E M=$ morte e morrer; $I N T=$ intimidade Fonte: elaboração da autora. 